\title{
Adult Onset Intestinal Malrotation: An Often Neglected Entity
}

Aidi Aswadi Halim Lim

International Islamic University of Malaysia

Introduction: Intestinal malrotation is a consequent of deviation from the normal 270 degrees counter-clockwise rotation of the midgut during embryogenesis. It is a typically paediatric disease that manifest itself in the first month of life, usually as midgut volvulus. However adult manifestation of intestinal malrotation is not unheard of, albeit rare. Case report: his is a case report of 46 year old man with chronic abdominal pain who was subsequently diagnosed with intestinal malrotation. He was initially referred for barium enema following an incomplete colonoscopy. The suspicion of intestinal malrotation arose during barium enema, as the large bowel loops were all located on the left side. He subsequently had a plain CT following the barium enema, which confirmed the suspicion. In view of this finding, the patient was offered Ladd's procedure by the surgical team. The patient duly declined and opted for conservative watchful waiting instead. 